\title{
iCAR: Intersection-based Connectivity Aware Routing in Vehicular Ad hoc Networks
}

\author{
Nizar Alsharif* ${ }^{*}$, Sandra Céspedes ${ }^{\dagger}$, and Xuemin (Sherman) Shen* \\ *Department of Electrical and Computer Engineering, University of Waterloo, Waterloo, Canada \\ $\ddagger$ Umm Al-Qura University, Makkah, Saudi Arabia \\ ${ }^{\dagger}$ Department of Information and Communications Technology, Icesi University, Cali, Colombia \\ \{nalsharif,slcesped,xshen\}@bbcr.uwaterloo.ca
}

\begin{abstract}
In this paper, we propose an intersection-based connectivity-aware routing protocol $(i \mathrm{CAR})$ for vehicular ad hoc networks (VANETs) to enable infotainment and interactive applications, as well as multi-hop Internet access in urban environments. $i$ CAR is a novel protocol that takes into consideration real-time vehicular traffic information and the experienced packet delivery delay per road, in order to improve the routing performance by dynamically selecting roads with a guaranteed connectivity and a reduced delivery delay. This is achieved by deploying a microscopic view of vehicles location to proactively estimate roads connectivity and the minimum link lifetime per road. Detailed analysis and simulation-based evaluations show that $i$ CAR significantly improves the network performance in terms of packet delivery ratio and end-to-end delay with a negligible cost of communication overhead.
\end{abstract}

\section{INTRODUCTION}

The evolution of wireless communications technologies has inspired governments, standardization organizations, academic researchers, and automotive manufacturers, to investigate and deploy the communication capabilities in order to increase transportation safety and efficiency, as well as drivers and passengers comfort. By integrating wireless communications devices into vehicles, vehicular networks enable a variety of applications and services for Intelligent Transportation Systems (ITS) and road users. These applications can be classified according to the number of hops traversed in the vehicular network into: 1) one-hop applications, such as disseminating a warning message about a slippery road to the nearby vehicles; and 2) multi-hop applications, such as Internet access, chatting, and interactive gaming between passengers and fixed points located at the infrastructure, or between passengers located in different areas of the city.

Vehicular ad hoc networks (VANETs) are a special class of the self-organized mobile ad hoc networks (MANETs), where vehicles equipped with on-board units (OBUs) can communicate with other vehicles (V2V communications), and with roadside units (RSUs) installed along roads (V2I communications). Routing is one of the vital mechanisms to achieve reliable multi-hop applications in VANETs. However, the highly dynamic VANET topology poses many challenges for designing an efficient multi-hop routing scheme. Vehicular communication projects such as CarTalk2000 [1] and NoW [2] have considered position-based routing (PBR) [3] to cope with the challenging VANET characteristics.
PBR requires each vehicle to periodically broadcast its geographic location in beacon packets. Fortunately, this requirement can be easily achieved in VANETs as each vehicle is equipped with a Global Positioning Service (GPS) receiver used to determine its location. In PBR, the routing of packets from source to destination does not depend on predetermined forwarders but rather the forwarders can be different for each packet exchanged between the same source and destination. Consequently, PBR adapts to the highly dynamic VANET topology. Studies have shown that PBR protocols perform better than traditional topology-based routing protocols in both urban and highway scenarios [3].

Numerous PBR protocols have been previously proposed in both MANET and VANET contexts [3], [4]. Lochert et al. introduce the anchor-based routing protocols GSR [5] and GPCR [6]. The protocols consider a set of anchors that are overlaid on top of the city map. In GSR, the route is attached to each packet by means of intersections information based on a static map, whereas in GPCR the routing decision is taken at each intersection, based on local information. A-STAR [7] is another anchor-based routing protocol that uses city buses paths to estimate road connectivity.

In GyTAR [8], routing decisions are taken at the intersections, based on the distance between the next intersection and the final destination of packets, together with the wireless connectivity to the next intersections. GyTAR also defines a procedure to collect traffic density information, and to estimate road connectivity based on collected information. On the other hand, E-GyTAR [9] considers vehicles movement direction by assigning a higher weight for vehicles heading to the target junction. Both GyTAR and E-GyTAR assign a higher weight to roads with high vehicular density, because those roads are more likely to have stable network connectivity. Chang et al. introduce STAR [10] to analyze the problem of network partitioning caused by variable densities appearing due to traffic lights. In STAR, authors propose detecting red lights by means of video cameras, and to give the priority to connected roads with red lights instead of using the rule of green-light-first.

Most of the routing protocols that consider traffic density in road selection give priority to dense roads. This eventually makes data routing converge to certain roads with high vehicular density, causing data traffic congestions. Moreover, recent 
studies show that vehicles may also act as communication obstacles [11], and a higher vehicular density can cause multiple transmission failures. When vehicles act as obstacles, the number of intermediate forwarders required to traverse a certain road increases. In addition, traffic lights prompt vehicles to be clustered at the end of roads with a probability of forming disconnected networks between clusters. Thus, a high vehicular density in a certain road does not necessarily guarantee network connectivity, but can cause a high delivery delay instead.

In this paper, we propose an intersection-based traffic aware routing protocol $(i \mathrm{CAR})$, which combines static map and realtime traffic information, in order to improve VANET performance in city scenarios. $i$ CAR calculates an adaptive lower bound of connectivity lifetime, which enables better routing decisions based on guaranteed connectivity information to the adjacent intersections, with a minimum cost of communication overhead. For each road, $i$ CAR takes into consideration both vehicular density and average communication delay. Thus, roads with high data volume and high vehicular density have a low preference to be selected as forwarding paths, in order to avoid an increased average transmission delay. As a result, a fair distribution of packets is achieved across the network, and the overall network performance can be improved.

The reminder of this paper is organized as follows. In Section II we described the VANET model, followed by a detailed description of $i$ CAR in Section III. Next, we present a simulation-based evaluation and discussion of the results in Section IV. The concluding remarks are presented in Section V.

\section{VANET MODEL}

The scenario under consideration consists of roads with intersections such as in an urban area. Roads vary in terms of width, length, and vehicular traffic density. Each intersection, or junction, has a unique ID $\left(I_{I D}\right)$. Roads between intersections are referred to as road segments and each road segment has a well-defined geographic boundary, which is referred to by its endpoint junctions $\left(I_{i} I_{j}\right)$.

A set of RSUs are available and moderately distributed in the city area to provide infrastructure and Internet access to vehicles, in addition to a wide range of ITS services. However, RSUs do not provide full communication coverage across the city, as it happens nowadays with WiFi hotspots or with in-deployment 802.11p vehicular networks. Each RSU has a unique ID $\left(R_{I D}\right)$. Vehicles, on the other hand, are equipped with OBUs for communications with other vehicles and with RSUs, and each OBU has a unique ID $\left(v_{I D}\right)$. Vehicles are also equipped with a GPS to obtain geographic location and velocity vectors, and to enable synchronization within the network. All vehicles have access to identical digital maps that contain information about road segments, junctions, and RSUs locations.

Packets are forwarded from/to vehicles and RSUs via multihop routing, in order to provide low cost (or free) Internet and information access. This model is widely accepted for infotainment multi-hop applications and Internet access [12], [13], [14]. Our scope in this paper is to consider the multihop routing part between vehicles, i.e., the route selection for a packet to be forwarded from its source to its destination via a number of intermediate mobile OBUs. Thus, we consider the routing from an RSU to an OBU, from an OBU to an RSU, and from an OBU to another OBU.

\section{III. $i$ CAR- INTERSECTION-BASED CONNECTIVITY-AWARE ROUTING IN VANETS}

$i$ CAR is a unicast PBR protocol designed for the multihop access to interactive and infotainment applications in city scenarios. Examples of these applications include the download of information from fixed stations (e.g., locationbased online advertisement) or from Internet gateways, as well as file sharing, chatting, and interactive gaming between mobile vehicles. To operate in the city, $i \mathrm{CAR}$ requires the availability of road map and location information of nodes, which is obtained from the GPS installed in vehicles. Location service is also required in order to obtain the location of a mobile destination; we consider such information to be available through existent location services, such as GLS [15].

$i$ CAR combines local real-time road condition information and static road-topology information extracted from digital maps. Real-time information is locally and dynamically calculated at each road, by sending out a control packet (CP) to discover connectivity and collect vehicular traffic information while traversing the road segment. CPs are probabilistically generated at each intersection to maintain updated connectivity information. Scores are assigned to each road segment, based on the volume of vehicular traffic in that road and the delay experienced by the associated CP. After that, the scores are disseminated locally in beacon packets exchanged by vehicles at the intersections. The beacons also inform the validity period of each score.

Two routing strategies are employed: next-junction selection and next-hop selection. Packets are forwarded from junctionto-junction based on the next-junction selection strategy, and forwarded hop-by-hop within roads based on the next-hop selection strategy.

We describe in detail the $i \mathrm{CAR}$ operation by introducing its four components as follows:

\section{A. Road Segment Evaluation (RSE)}

RSE is a heuristic distributed approach aimed at evaluating the suitability of road segments for the forwarding of packets. It also maintains a global parameter that enables the fair and accurate distribution of packets. RSE procedure is carried out by a vehicle $v_{i}$ entering to a road segment $A B . v_{i}$ triggers the RSE with probability $P$, where $P$ is a function of the road segment conditions and the remaining lifetime of the road score $Q_{A B}$. When RSE is triggered, $v_{i}$ transmits a unicast discovery packet $(\mathrm{CP})$ to the center of the next road intersection. CP is forwarded hop-by-hop according to the next-hop selection strategy. Fig. 1 shows the lightweight packet format of $\mathrm{CP}$. Each forwarder involved in the tranmission of CP (including $v_{i}$ ) accumulates the number of vehicles located between itself 


\begin{tabular}{|l|l|}
\hline Originator: Vebicle_ID & ToG: Timestamp \\
\hline From: Junction_ID & To: Junction_ID \\
\hline NTotal: Total Number of Vebicles \\
\hline Lifetime: Minimum Link Lifetime \\
\hline \multicolumn{2}{|l|}{ Total number of intermediate hops } \\
\hline
\end{tabular}

Fig. 1. RSE Control Packet (CP) Fields

and the vehicle chosen as the next forwarder. Such a number is stored in the $N_{\text {total }}$ field. The origination time and the number of hops $h$ are also recorded in CP. Before sending the packet to the next hop, the forwarder runs the Validity Period Calculation (VPC) algorithm (described later in Section III-B) and updates the lifetime field if it has a shorter estimated link lifetime.

When CP reaches the next intersection, the closest vehicle to the center of the intersection, say $v_{j}$, is responsible of generating the updated score $Q_{A B} . v_{j}$ then announces the score across the intersection, and sends it back to the location where the RSE procedure was triggered. $Q_{A B}$ is calculated by $v_{j}$ as follows:

$$
Q_{A B}=\alpha_{1} \cdot \min \left(1, \frac{N_{a v g}}{N_{c o n}}\right)+\alpha_{2} \cdot\left(\frac{T}{t_{a v g}}\right)+\alpha_{3} \cdot\left(\frac{h_{m i n}}{h}\right),
$$

where $N_{a v g}$ is the average number of vehicles per one hop transmission distance, $N_{c o n}$ is a constant representing the average number of vehicles per one hop transmission distance, based on statistics of city scenarios, $T$ is the minimum onehop transmission delay (i.e., the delay of transmitting a similar packet with no buffering delay and perfect channel conditions), $t_{\text {avg }}$ is the average per hop transmission delay of the CP, $h_{\text {min }}$ is the minimum number of hops required to traverse the road segment, $h$ is the number of hops actually traversed from $v_{i}$ to $v_{j}$, and $\alpha_{1}, \alpha_{2}$ and $\alpha_{3}$ are weighting factors for the vehicular density, the one-hop transmission delay, and the number of intermediate forwarders, respectively.

The delivery of $\mathrm{CP}$ at the next intersection indicates the instantaneous connectivity of the road. The information stored in $\mathrm{CP}$ helps the vehicle at the target intersection to assign a road score with a validity period (or lifetime) for such a score. As shown in (1), the effect of the vehicular density on the score is upper-bounded by $\alpha_{1}$, and $N_{a v g}$ is calculated as follows:

$$
N_{a v g}=\frac{N_{t o t a l}}{h} .
$$

The average delay per hop indicates the delay due to both queuing in the forwarders' buffers and retransmissions. $t_{\text {avg }}$ is calculated as follows:

$$
t_{\text {avg }}=\frac{\left(t_{2}-t_{1}\right)}{h}
$$

where $t_{2}$ and $t_{1}$ are the reception time of $\mathrm{CP}$ at the target intersection and its originating time, respectively.

As mentioned before, vehicles with variable dimensions may work as obstacles for transmission, and may reduce the effective transmission range in their vicinity [11]. A large number of obstructing vehicles results in shorter effective transmission ranges, which derives in a higher number of intermediate transmissions. $i$ CAR reduces the score for road segments with relatively high number of intermediate forwarders, as shown in (1). The minimum number of forwarders, $h_{m i n}$, is calculated as follows:

$$
h_{\min }=\lceil l / R\rceil,
$$

where $l$ is the road segment length and $R$ is the transmission range.

When $v_{i}$ triggers the RSE procedure, it sets a timer $T_{\max }$ and waits for reception of the returning $Q_{A B}$ or another $\mathrm{CP}$ coming from the other side. If $v_{i}$ does not receive such information before the timer expires, then $v_{i}$ sets the score to zero. If a forwarder does not find a next-hop during the forwarding of $\mathrm{CP}$, it sends the $\mathrm{CP}$ back to the originator with an indication of road disconnection. $Q_{A B}$ is also set to zero in such a case. The $Q_{A B}$ is announced across the intersection and a random validity period (RBP), which works as a backoff period, is set to prevent multiple CP transmissions.

The probability $P$ that $v_{i}$ triggers the RSE procedure when entering the road segment is designed in such a way that the score, $Q_{A B}$, is refreshed when it has a long validity period, and to allow re-computing the value before the current validity expires. Since $i$ CAR considers not only the road segment connectivity, but also the packet delivery delay at the moment of $Q_{A B}$ calculation, the renewing of $Q_{A B}$ before the expiration time is beneficial. In (5), we present one way to calculate $P$, where $t_{r e m}$ is the remaining validity period and $C$ is a constant. To ensure the renewing of $Q_{A B}$ before the validity expires, $C$ is related to the expected time required to traverse the particular road segment when performing the RSE procedure.

$$
P= \begin{cases}e^{-\frac{t_{r e m}-C}{2}}, & t_{r e m} \geq C \\ 1 & t_{r e m}<C\end{cases}
$$

\section{B. Validity Period Calculation (VPC)}

The goal of VPC is to define a lower bound for the connectivity lifetime of a given road segment. In other words, it aims at predicting the time at which a disconnection may occur. By using local information stored by the $\mathrm{CP}$ forwarders in the routing table, $i$ CAR performs the VPC algorithm described in Fig. 2. Once VPC is executed, it is possible to assign a validity period for each score associated with a successful CP delivery.

In Fig. 2, each $\mathrm{CP}$ forwarder estimates the time required for the first link breakage in the area between itself and the destination junction of $\mathrm{CP}$ that falls within its transmission range. This zone is called the area of interest $(A o I)$ of the forwarder, as illustrated in Fig. 3. A link breakage in the AoI is detected at the time when less than one node is present in the AoI. In order to perform this detection, the forwarder employs local information, e.g., positions, velocities, and directions of neighboring vehicles. VPC divides the vehicles within $A o I$ 


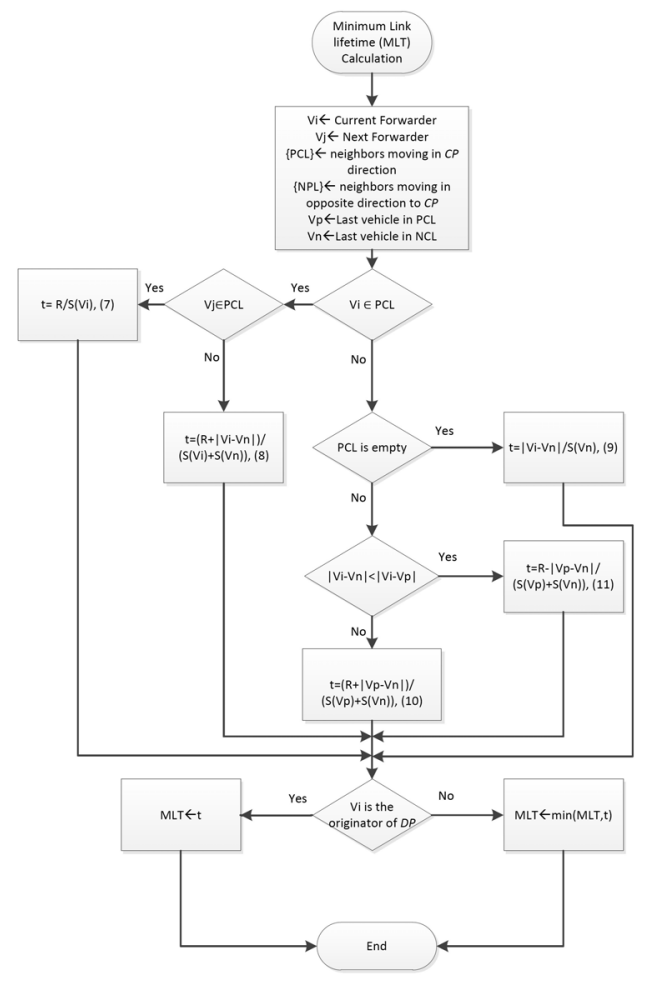

Fig. 2. VPC Algorithm

into two clusters. The cluster of vehicles moving in the same direction in which $\mathrm{CP}$ is being forwarded, called the positive cluster (PCL), and the cluster of vehicles moving in the opposite direction, called the negative cluster (NCL).

The vehicle at the tail of each cluster is identified, so that the tail of the PCL is referred as $v_{p}$, and the tail of the NCL is referred as $v_{n}$. According to Fig. 2, each CP forwarder calculates the link lifetime in its $A o I$ based on one of the following cases:

a. Current forwarder, $v_{i}$, and next forwarder, $v_{j}$ are both in PCL: In this case, the first link breakage is predicted to happen at the time when $v_{i}$ leaves the zone previously defined by $A o I$.

b. The current forwarder $v_{i}$ is in PCL and the next forwarder $v_{j}$ is in NCL: A disconnection may happen when $v_{i}$ and $v_{n}$ move out of each other's transmission range.

c. Current forwarder $v_{i}$ and next forwarder $v_{j}$ are both in NCL, and PCL is an empty set: The disconnection may occur when $v_{n}$ leaves the $A o I$.

d. The current forwarder $v_{i}$ is in NCL, and PCL is not an empty set: When $v_{n}$ and $v_{p}$ are approaching each other, the minimum estimated link lifetime is the time for these vehicle to reach and then move away from each other's transmission range. On the other hand, if $v_{n}$ and $v_{p}$ are already moving away from each other, the estimated link lifetime is the time required for them to be out of each other's transmission range.

We consider that $R$ is much larger than the road width, thus, we neglect the effect of vehicles located in multiple lanes and

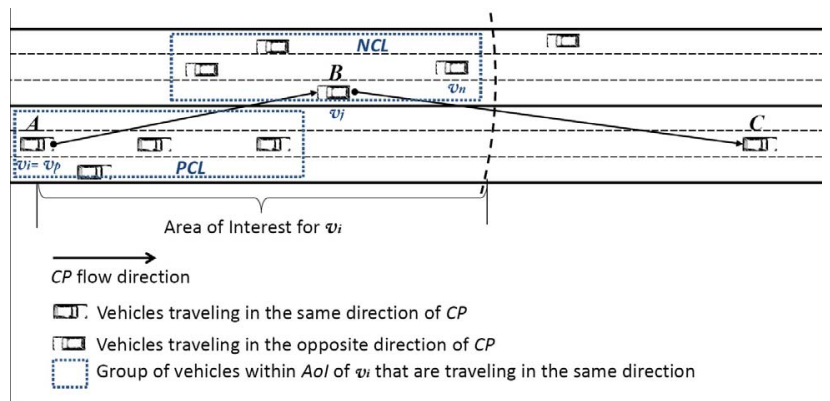

Fig. 3. Example of VPC operation at the current CP forwarder $\left(v_{i}\right)$

assume they all move in one dimension. Equations (7) to (11) in Fig. 2 describe the aforementioned cases. Note that $\left|v_{i}-v_{j}\right|$ denotes the absolute distance between vehicle $v_{i}$ and $v_{j}$.

The calculated lifetime is upper-bounded by the time required by the forwarder $\left(v_{i}\right)$, to drive for $\mathrm{R}$ meters in the same direction that $\mathrm{CP}$ is being forwarded, i.e., $t_{\max }=R / S\left(v_{i}\right)$, where $R$ is the forwarder transmission radius and $S\left(v_{i}\right)$ is the speed of $v_{i}$ (for simplicity we assume that neighboring vehicles moving in the same direction are all moving with the same speed). The score lifetime for the entire road segment would be the minimum lifetime of all the lifetimes calculated by each forwarder. This value is updated and recorded in CP before being forwarded at each hop.

\section{Next-junction selection}

When a data packet reaches an intersection, the next junction is selected based on the adjacent intersection scores, the geographic location of the intersections, and the packet's final destination location. The routing header of the packet is updated accordingly. The next junction is selected to be the one with the highest scores according to the following formula:

$$
S\left(I_{j}\right)=B_{1} \cdot\left(1-\frac{D_{j}}{D_{i}}\right)+B_{2} \cdot\left(Q\left(I_{i}, I_{j}\right)\right) .
$$

The first component in (6) is the progression toward the destination, where $D_{j}$ denotes the driving distance from the adjacent junction $j$ to the destination, and $D_{i}$ denotes the driving distance from the current junction $i$ to the destination. The second component is the road segment score for the road between $i$ and $j . B_{1}$ and $B_{2}$ are weighting factors for each component.

In this way, $i$ CAR adopts a distributed anchor-based routing where data packets are routed from intersection to intersection based on real-time road condition information. Roads scores are updated periodically and dynamically via the RSE procedure, and exchanged via beacon messages.

\section{Next-hop selection}

$i$ CAR employs a greedy-based next-hop selection to choose the next forwarder for a packet being transmitted between two junctions. The location of neighboring vehicles is known by means of the beacon packets; however, vehicles may move out of each other's transmission range during an inter-beacon interval, which in turn causes wrong routing decisions and 
retransmissions. This problem can be avoided by predicting the existence of available forwarders based on the last reports about neighbors' positions and speeds [8]. Moreover, beacon packets may include RSSI information about neighbors, which reflect the status of signal quality and potential interference. In addition to beacons, RSSI information can be refreshed by RTS, CTS, and other data packets. $i$ CAR selects the next-hop from the set of neighbors that are predicted to be within the communication range of the current forwarder, and that have a strong RSSI. If the algorithm fails to find a forwarder with such a strategy, the recovery strategy store-carry-and-forward is employed instead.

\section{Evaluation}

In this section, we present a simulation-based evaluation of $i$ CAR. Our protocol is compared with the implementations of GPSR [16] and GyTAR [8]. GPSR is a basic PBR protocol commonly employed for performance benchmarks. GyTAR is a recent PBR protocol and one of the most closely related protocols to our work.

\section{A. Simulation Setting}

We have implemented a simulation for VANETs in Matlab. The environment includes a digital city map with a grid area of $7000 \mathrm{~m} \times 7000 \mathrm{~m}$ and bidirectional roads. Roads vary in terms of number of lanes: bidirectional lanes with lower vehicular traffic to represent residential areas, and roads with two to four lanes per direction to represent main connecting city roads. A total of 165 intersections with 45 controlled intersections have been included, and two different average vehicular densities ( 6 and 12 vehicles/lane/ $\mathrm{Km}$ ) are employed to represent low and high vehicular traffic volumes.

The system and simulation parameters for the operation of $i$ CAR are described in Table I. GyTAR and GPSR parameters are set according to [8] and [16], respectively. We do not consider retransmissions caused by collision of packets. Nodes implement a FIFO packet queue, such as the AC queues designed for WAVE's MAC layer [17], to buffer packets pending for transmission. A free space model with urban area path loss exponent is deployed to estimate the RSSI [18]. Besides attenuation, we marked $5 \%$ of the vehicles as obstructing vehicles, and $P_{L O S}$ is calculated according to the model presented in [17]. The path loss exponent is then chosen to be LOS or non-LOS, depending on the $P_{L O S}$ value [18].

\section{B. Simulation Results and Analysis}

The performance metrics employed to compare and evaluate the proposed protocol are: packet delivery ratio (PDR), packet delivery delay, and routing overhead. The simulation results and discussion are presented as follows.

1) Packet Delivery Ratio: The PDR is the average ratio of packets received to packets sent. Fig. 4a shows that $i$ CAR outperforms both GyTAR and GPSR. iCAR and GyTAR, which are anchor-based, have significantly higher PDR than GPSR, due in part to the prediction of the existence of neighbors before transmitting packets. $i$ CAR and GyTAR rely on the existence of vehicular traffic in order to consider a road

\begin{tabular}{|l|l|l|l|}
\hline Parameter & Value & Parameter & Value \\
\hline$\alpha_{1}$ & 0.333 & $\mathcal{B}_{1}$ & 0.5 \\
\hline$\alpha_{2}$ & 0.333 & $\mathcal{B}_{2}$ & 0.5 \\
\hline$\alpha_{3}$ & 0.333 & Simulation Duration & $30 \mathrm{sec}$ \\
\hline$N_{\text {con }}$ & 6 Vebicles & Inter-beacon Period & $500 \mathrm{msec}$ \\
\hline$T$ & $0.3 \mathrm{msec}$ & Transmission Range & $250 \mathrm{~m}$ \\
\hline$T_{\max }$ & $2 m s e c \times h_{\min }$ & Routing Protocol & $\begin{array}{l}\text { GPSR- } \\
\text { GyTAR-iCAR }\end{array}$ \\
\hline$C$ & & Packet Size & 512 byte \\
\hline$R B P$ & $1-5 \mathrm{sec}$ & Transmission Rate & $12 \mathrm{Mbps}$ \\
\hline$R S S I$ threshold & $0.6 \times$ RSSI $_{\max }$ & Packet lifetime & $500 \mathrm{msec}$ \\
\hline
\end{tabular}

TABLE I

Simulation PARAMETERS

for the forwarding of packets. GPSR instead, frequently resorts to the recovery strategy, which results in a larger number of traversed hops and in the dropping of packets before they reach the destination.

Furthermore, $i \mathrm{CAR}$ achieves nearly $15 \%$ increase of PDR with respect to GyTAR. This is mainly because $i$ CAR deploys a deterministic algorithm to trigger the RSE procedure. Thus, it is expected for $i$ CAR to always have deterministic connectivity information of the adjacent roads. On the other hand, GyTAR triggers the road connectivity evaluation procedure only when one of the cell leaders reaches the center of an adjacent intersection. Therefore, GyTAR's PDR is affected by traffic lights and controlled intersections: since vehicles are clustered at the road end-points during red lights, road disconnection occurs before the procedure to re-calculate the road connectivity score is triggered. In addition, the greedy routing and the convergence of packets on certain roads that have high vehicular traffic, as well as the buffering of packets during store-carry-and-forward, cause GyTAR to have multiple transmission failures, retransmissions, and high delivery delay, which eventually leads to packet losses.

2) Packet Delivery Delay: The packet delivery delay refers to the average end-to-end packet delay. Fig. 4b illustrates the average end-to-end packet delivery delay obtained from simulations by employing different packet generation rates. $i$ CAR shows to have the lowest packet delivery delay among the compared protocols. Unlike GyTAR, which considers the large volume of vehicular traffic at a certain road as a positive condition, $i$ CAR takes into consideration the actual delay required to traverse that road. Thus, alternative connected roads with less vehicular traffic and less experienced delay are also considered for packet delivery. Moreover, $i$ CAR's RSE procedure deterministically guarantees the connectivity of the road for a minimum period of time, which helps forwarders at intersections to make effective routing decisions. In this way, $i$ CAR minimizes the use of store-carry-andforward strategy. On the other hand, packets forwarded with GyTAR are frequently delayed when employing the storecarry-and-forward strategy. 


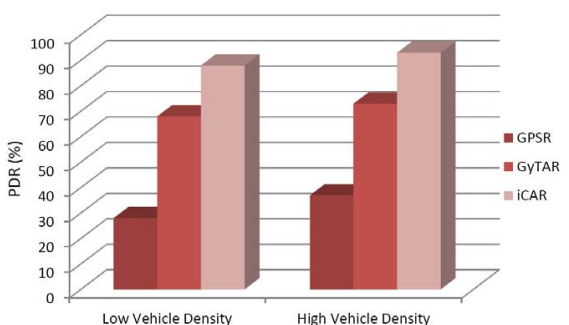

(a) Packet Delivery Ratio

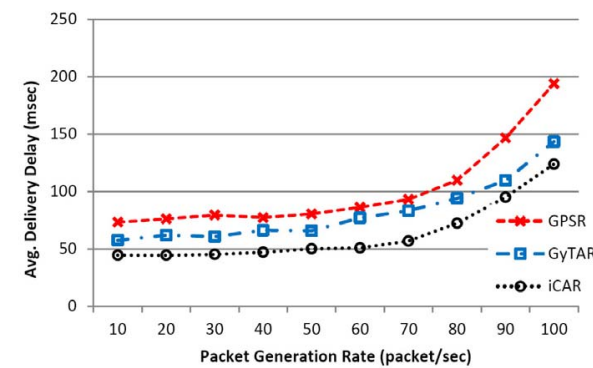

(b) Packet Delivery Delay

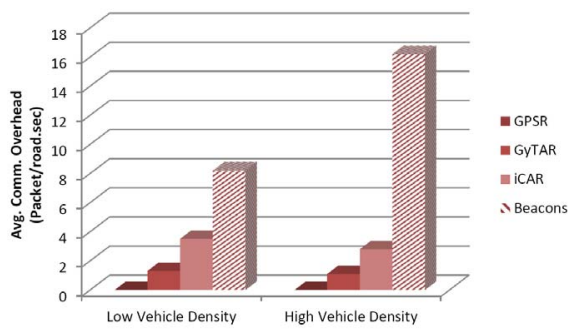

(c) Communication Overhead

Fig. 4. $i$ CAR Evaluation and Comparison

3) Routing Overhead: In general, PBR protocols have less communication overhead than traditional reactive routing protocols, because they do not employ route discovery and maintenance control messages for every flow of packets. On the other hand, beacon packets are the main communication overhead for PBR protocols. GyTAR and $i$ CAR introduce additional overhead when discovery packets are used to collect vehicular information along road segments. However, the frequency for generating such packets is much lower than the beaconing frequency, and the unicast nature of these discovery packets makes the introduced overhead almost negligible when compared to overhead caused by beacon packets.

Fig. $4 \mathrm{c}$ shows the average control packets sent per second on each road. The results indicate that the average beaconing overhead is the same for the different routing protocols; however, $i$ CAR has a higher average of discovery packets sent compared with GyTAR, which indicates that our protocol triggers more frequently the road segment evaluation. Nonetheless, it is observed that with a higher vehicular density, the number of discovery packets is noticeably reduced. This is because $i$ CAR relates the RSE calls with the score validity period, as shown in (5). In both cases, the number of discovery packets is small and almost negligible.

\section{CONCLUSIONS AND FUTURE WORK}

We have proposed $i \mathrm{CAR}$, a position-based routing protocol that improves the VANETs routing performance in dense city scenarios, by adjusting the next-junction selection procedure based on real-time traffic, the delay information for each road, and a deterministic connectivity lifetime estimation. Simulation results have demonstrated that $i$ CAR outperforms other position-based routing protocols, such as GPSR and GyTAR, in terms of higher packet delivery ratio and reduced packet delivery delay, with a negligible communication overhead. To refine $i$ CAR, our future work will focus on sensitivity analysis to adjust the different system parameters of $i \mathrm{CAR}$ in order to optimize its performance. In addition, as junction scores are calculated with scalable variables, e.g., independent of road length, we will consider the study of an infrastructure-based protocol, where scores are reported to a central routing entity via distributed RSUs, in order to obtain global network view and optimal end-to-end routing.

\section{REFERENCES}

[1] D. Reichardt, M. Miglietta, L. Moretti, P. Morsink, and W. Schulz, "CarTALK 2000: safe and comfortable driving based upon inter-vehiclecommunication," in Proc. IEEE IV'02, vol. 2, 2002, pp. 545-550.

[2] A. Festag, G. Noecker, M. Strassberger, A. Lübke, B. Bochow, M. Torrent-Moreno, S. Schnaufer, R. Eigner, C. Catrinescu, and J. Kunisch, "NoW - Network on Wheels: Project Objectives, Technology and Achievements," in Proc. WIT, 2008.

[3] F. Li and Y. Wang, "Routing in vehicular ad hoc networks: A survey," IEEE Veh. Technol. Mag., vol. 2, no. 2, pp. 12-22, Jun. 2007.

[4] C. A. T. H. Tee and A. C. R. Lee, "Survey of position based routing for Inter Vehicle Communication system," in Proc. DFmA, Oct. 2008, pp. $174-182$.

[5] C. Lochert, H. Hartenstein, J. Tian, H. Fussler, D. Hermann, and M. Mauve, "A routing strategy for vehicular ad hoc networks in city environments," in Proc. IEEE IV'03, 2003, pp. 156-161.

[6] C. Lochert, M. Mauve, H. Fussler, and H. Hartenstein, "Geographic routing in city scenarios," SIGMOBILE Mob. Comput. Commun. Rev., vol. 9, no. 1, pp. 69-72, 2005.

[7] B.-c. Seet, G. Liu, B.-s. Lee, C.-h. Foh, and K.-k. Lee, "A-STAR: A mobile ad hoc routing strategy for metropolis vehicular communications," Proc. NETWORKING, pp. 989 - 999, 2004.

[8] M. Jerbi, S.-M. Senouci, T. Rasheed, and Y. Ghamri-Doudane, "Towards Efficient Geographic Routing in Urban Vehicular Networks," IEEE Trans. Veh. Technol., vol. 58, no. 9, pp. 5048-5059, Nov. 2009.

[9] S. Bilal, S. Madani, and I. Khan, "Enhanced Junction Selection Mechanism for Routing Protocol in VANETs," Int. Arab J. Inf. Technol., vol. 8, no. 4, pp. 422-429, 2011.

[10] J.-J. Chang, Y.-H. Li, W. Liao, and I.-C. Chang, "Intersection-based routing for urban vehicular communications with traffic-light considerations," IEEE Wireless Commun., vol. 19, no. 1, pp. 82-88, Feb. 2012.

[11] M. Boban, T. T. V. Vinhoza, M. Ferreira, J. Barros, and O. K. Tonguz, "Impact of Vehicles as Obstacles in Vehicular Ad Hoc Networks," IEEE J. Sel. Areas Commun., vol. 29, no. 1, pp. 15-28, Jan. 2011.

[12] C. Zhang, X. Lin, R. Lu, P.-H. Ho, and X. Shen, "An Efficient Message Authentication Scheme for Vehicular Communications," IEEE Trans. Veh. Technol., vol. 57, no. 6, pp. 3357-3368, Nov. 2008.

[13] S. Céspedes, N. Lu, and X. Shen, "VIP-WAVE: On the Feasibility of IP Communications in $802.11 \mathrm{p}$ Vehicular Networks," IEEE Trans. Intell. Transp. Syst., to appear, pp. 1-16, 2012.

[14] N. Alsharif, A. Wasef, and X. Shen, "ESPR: Efficient Security Scheme for Position-Based Routing in Vehicular Ad Hoc Networks," in Proc. IEEE GLOBECOM, Dec. 2010, pp. 1-5.

[15] J. Li, J. Jannotti, D. S. J. De Couto, D. R. Karger, and R. Morris, “A scalable location service for geographic ad hoc routing," in Proc. ACM MobiCom, Aug. 2000, pp. 120-130.

[16] B. Karp and H. T. Kung, "GPSR: greedy perimeter stateless routing for wireless networks," in Proc. ACM MobiCom, 2000, pp. 243-254.

[17] C. Suthaputchakun and Z. Sun, "Routing protocol in intervehicle communication systems: a survey," IEEE Commun. Mag., vol. 49, no. 12, pp. 150-156, Dec. 2011.

[18] H. Xia, "A simplified analytical model for predicting path loss in urban and suburban environments," IEEE Trans. Veh. Technol., vol. 46, no. 4, pp. 1040-1046, 1997. 